\title{
On some properties of the generalized Mittag-Leffler function
}

\author{
Mumtaz Ahmad Khan and Shakeel Ahmed
}

\begin{abstract}
This paper deals with the study of a generalized function of Mittag-Leffler type. Various properties including usual differentiation and integration, Euler(Beta) transforms, Laplace transforms, Whittaker transforms, generalized hypergeometric series form with their several special cases are obtained and relationship with Wright hypergeometric function and Laguerre polynomials is also established.
\end{abstract}

2000 Mathematics Subject Classification: 33C45, 47G20, 26A33.

Keywords: Mittag-Leffler function; Generalized hypergeometric function; Fox's H function

\section{Introduction}

In 1903, the Swedish mathematician Gosta MittagLeffler (1903) introduced the function

$$
E_{\alpha}(z)=\sum_{n=0}^{\infty} \frac{z^{n}}{\Gamma(\alpha n+1)}
$$

where $z$ is a complex variable and $\Gamma$ is a Gamma function $\alpha \geq 0$. The Mittag-Leffler function is a direct generalisation of exponential function to which it reduces for $\alpha=1$. For $0<\alpha<1$ it interpolates between the pure exponential and hypergeometric function $\frac{1}{1-z}$. Its importance is realized during the last two decades due to its involvement in the problems of physics, chemistry, biology, engineering and applied sciences. Mittag-Leffler function naturally occurs as the solution of fractional order differential or fractional order integral equation.

The generalisation of $E_{\alpha}(z)$ was studied by Wiman (1905) in 1905 and he defined the function as

$$
E_{\alpha, \beta}(z)=\sum_{n=0}^{\infty} \frac{z^{n}}{\Gamma(\alpha n+\beta)}, \quad(\alpha, \beta \in \mathbb{C}, \operatorname{Re}(\alpha)>0, \operatorname{Re}(\beta)>0)
$$

which is known as Wiman function.

*Correspondence: shakeelamu81@gmail.com

Department of Applied Mathematics, Faculty of Engineering, Aligarh Muslim University, Aligarh-202002, India
In 1971, Prabhakar (1971) introduced the function $E_{\alpha, \beta}^{\gamma}(z)$ in the form of

$$
\begin{aligned}
E_{\alpha, \beta}^{\gamma}(z)= & \sum_{n=0}^{\infty} \frac{(\gamma)_{n} z^{n}}{\Gamma(\alpha n+\beta) n !},(\alpha, \beta, \gamma, \in \mathbb{C}, \operatorname{Re}(\alpha)>0, \\
& \operatorname{Re}(\beta)>0, \operatorname{Re}(\gamma)>0),
\end{aligned}
$$

where $(\gamma)_{n}$ is the Pochhammer symbol (Rainville (1960))

$$
\begin{aligned}
& (\gamma)_{n}=\frac{\Gamma(\gamma+n)}{\Gamma(\gamma)},(\gamma)_{0}=1, \\
& (\gamma)_{n}=\gamma(\gamma+1)(\gamma+2) \cdots(\gamma+n-1), n \geq 1 .
\end{aligned}
$$

In 2007, Shukla and Prajapati (2007) introduced the function $E_{\alpha, \beta}^{\gamma, q}(z)$ which is defined for $\alpha, \beta, \gamma \in \mathbb{C} ; \operatorname{Re}(\alpha)>$ $0, \operatorname{Re}(\beta)>0, \operatorname{Re}(\gamma)>0$ and $q \in(0,1) \bigcup \mathbb{N}$ as

$$
E_{\alpha, \beta}^{\gamma, q}(z)=\sum_{n=0}^{\infty} \frac{(\gamma)_{q n} z^{n}}{\Gamma(\alpha n+\beta) n !}
$$

In 2009, Tariq O. Salim (2009) introduced the function the function $E_{\alpha, \beta}^{\gamma, \delta}(z)$ which is defined for $\alpha, \beta, \gamma, \delta \in \mathbb{C}$; $\operatorname{Re}(\alpha)>0, \operatorname{Re}(\beta)>0, \operatorname{Re}(\gamma)>0, \operatorname{Re}(\delta)>0$ as

$$
E_{\alpha, \beta}^{\gamma, \delta}(z)=\sum_{n=0}^{\infty} \frac{(\gamma)_{n} z^{n}}{\Gamma(\alpha n+\beta)(\delta)_{n}}
$$

\section{Springer}


In 2012, a new generalization of Mittag-Leffler function was defined by Salim (2012) as

$$
E_{\alpha, \beta, p}^{\gamma, \delta, q}(z)=\sum_{n=0}^{\infty} \frac{(\gamma)_{q n} z^{n}}{\Gamma(\alpha n+\beta)(\delta)_{p n}}
$$

where $\alpha, \beta, \gamma, \delta \in \mathbb{C} ; \min (\operatorname{Re}(\alpha), \operatorname{Re}(\beta), \operatorname{Re}(\gamma), \operatorname{Re}(\delta))>0$

In this paper a new definition of generalized Mittag-Leffler function is investigated and defined as

$$
E_{\alpha, \beta, \delta}^{\gamma, q}(z)=\sum_{n=0}^{\infty} \frac{(\gamma)_{q_{n}} z^{n}}{\Gamma(\alpha n+\beta)(\delta)_{n}}, \alpha, \beta, \gamma, \delta \in \mathbb{C},
$$

where

$\min (\operatorname{Re}(\alpha), \operatorname{Re}(\beta), \operatorname{Re}(\gamma), \operatorname{Re}(\delta)>0)$ and $\mathrm{q} \in(0,1) \bigcup \mathbb{N}$,

Further the generalization of definition (1.7) is investigated and defined as follows

$$
E_{\alpha, \beta, \nu, \sigma, \delta, p}^{\mu, \rho, \gamma, q}(z)=\sum_{n=0}^{\infty} \frac{(\mu)_{\rho n}(\gamma)_{q n} z^{n}}{\Gamma(\alpha n+\beta)(v)_{\sigma n}(\delta)_{p n}},
$$

where,

$$
\alpha, \beta, \gamma, \delta, \mu, \nu, \rho, \sigma \in \mathbb{C} ; p, q>0 \text { and } q \leq \operatorname{Re}(\alpha)+p,
$$

and

$m i n(\operatorname{Re}(\alpha), \operatorname{Re}(\beta), \operatorname{Re}(\gamma), \operatorname{Re}(\delta), \operatorname{Re}(\mu), \operatorname{Re}(\nu), \operatorname{Re}(\rho), \operatorname{Re}(\sigma))>0$

The definition (1.9) is a generalization of all above functions defined by (1.1)-(1.7).

- Setting $\mu=v, \rho=\sigma$, it reduces to Eq. (1.6) defined by Salim (2012), in addition of that if $p=1$, it reduces to Eq. (1.7).

- Setting $\mu=v, \rho,=\sigma$ and $p=q=1$, it reduces to Eq. (1.5) defined by Salim (2009).

- Setting $\mu=v, \rho=\sigma$ and $p=\delta=1$, it reduces to Eq. (1.4) defined by Shukla and Prajapati (2007), in addition of that if $q=1$, then we get Eq. (1.3) defined by Prabhakar (1971).

- Setting $\mu=v, \rho=\sigma$ and $p=q=\delta=1$, it reduces to Eq. (1.2) defined by Wiman (1905), moreover if $\beta=1$, Mittag-Leffler function $E_{\alpha}(z)$ will be the result.

Throughout this investigation, we need the following well-known facts to study the various properties and relation formulas of the function $E_{\alpha, \beta, \nu, \sigma, \delta, p}^{\mu, \rho, \gamma, q}(z)$.
- Beta(Euler) transforms (Sneddon (1979)) of the function $f(z)$ is defined as

$$
B\{f(z) ; a, b\}=\int_{0}^{1} z^{a-1}(1-z)^{b-1} f(z) d z
$$

- Laplace transforms (Sneddon (1979)) of the function $f(z)$ is defined as

$$
L\{f(z) ; s\}=\int_{0}^{\infty} e^{-s z} f(z) d z
$$

- Mellin- transforms of the function $f(z)$ is defined as

$$
M\{f(z) ; s\}=f^{*}(s)=\int_{0}^{\infty} z^{s-1} f(z) d z, \quad \operatorname{Re}(\mathrm{s})>0,
$$

and the inverse Mellin-transform is given by

$$
f(z)=M^{-1}\left\{f^{*}(s) ; z\right\}=\frac{1}{2 \pi i} \int_{L} f^{*}(s) z^{-s} d s
$$

- Whittaker transform (Whittaker and Watson (1962))

$$
\int_{0}^{\infty} e^{\frac{-t}{2}} t^{\nu-1} W_{\lambda, \mu}(t) d t=\frac{\Gamma\left(\frac{1}{2}+\mu+v\right) \Gamma\left(\frac{1}{2}-\mu+v\right)}{\Gamma(1-\lambda+v)},
$$

where $\operatorname{Re}(\mu \pm v)>\frac{-1}{2}$ and $W_{\lambda, \mu}(t)$ is the Whittaker confluent hypergeometric function.

- The generalized hypergeometric function (Rainville (1960)) is defined as

$$
\left.{ }_{p} F_{q}\left[(\alpha)_{1}, \ldots,(\alpha)_{p} ;(\beta)_{1}, \ldots,(\beta)_{q} ; z\right)\right]=\sum_{n=0}^{\infty} \frac{\prod_{i=1}^{p}\left(\alpha_{i}\right)_{n}}{\left.\prod_{j=1}^{q}(\beta)_{j}\right)_{n}} \frac{z^{n}}{n !}
$$

- Wright generalized hypergeometric function (Srivastava and Manocha (1984)) is defined as

$$
\begin{aligned}
{ }_{p} \Psi_{q} & {\left[\left(a_{1}, A_{1}\right), \ldots,\left(a_{p}, A_{p}\right) ;\left(b_{1}, B_{1}\right), \ldots,\left(b_{q}, B_{q}\right) ; z\right] } \\
= & \sum_{n=0}^{\infty} \frac{\prod_{i=1}^{p} \Gamma\left(a_{i}+A_{i} n\right)_{n}}{\prod_{j=1}^{q} \Gamma\left(b_{j}+B_{j} n\right)} \frac{z^{n}}{n !}
\end{aligned}
$$

- Fox's H-function (Saigo and Kilbas (1998)) is given as

$$
\begin{gathered}
H_{p, q}^{m, n}\left[z \mid\left(a_{1}, \alpha_{1}\right), \ldots,\left(a_{p}, \alpha_{p}\right) ;\left(b_{1}, \beta_{1}\right), \ldots,\left(b_{q}, \beta_{q}\right)\right] \\
=\frac{1}{2 \pi i} \int_{L} \frac{\prod_{j=1}^{m} \Gamma\left(b_{i}+\beta_{i} s\right) \prod_{j=1}^{n} \Gamma\left(1-a_{i}-\alpha_{i} s\right)}{\prod_{j=m+1}^{q} \Gamma\left(1-b_{j}-\beta_{j} s\right) \prod_{j=n+1}^{p} \Gamma\left(a_{j}+\alpha_{j} s\right)} z^{-s} d s
\end{gathered}
$$

- Generalized Laguerre polynomials (Rainville (1960)). These are also known as Sonine polynomials and are defined as

$$
L_{n}^{(\alpha)}(x)=\frac{(1+\alpha)_{n}}{n !}{ }_{1} F_{1}[-n ; 1+\alpha ; x]
$$


- Incomplete Gamma function (Rainville (1960)). This is denoted by $\gamma(\alpha, z)$ and is defined by

$$
\gamma(\alpha, z)=\int_{0}^{z} e^{-t} t^{\alpha-1} d t, \operatorname{Re}(\alpha)>0,
$$

\section{Basic properties of the function $E_{\alpha, \beta, v, \sigma, \delta, p}^{\mu, \rho, \gamma, q}(z)$}

As a consequence of definitions (1.1)-(1.9) the following results hold:

Theorem 2.1. If $\alpha, \beta, \gamma, \delta, \mu, v, \rho, \sigma \in \mathbb{C}, \operatorname{Re}(\alpha)>$ $0, \operatorname{Re}(\beta)>0, \operatorname{Re}(\gamma)>0, \operatorname{Re}(\delta)>0, \operatorname{Re}(\mu)>0, \operatorname{Re}(v)>$ $0, \operatorname{Re}(\rho)>0, \operatorname{Re}(\sigma)>0$ and $p, q>0$ and $q \leq \operatorname{Re}(\alpha)+p$, then

$$
E_{\alpha, \beta, \nu, \sigma, \delta, p}^{\mu, \rho, \gamma, q}(z)=\beta E_{\alpha, \beta+1, v, \sigma, \delta, p}^{\mu, \rho, \gamma, q}(z)+\alpha z \frac{d}{d z} E_{\alpha, \beta+1, v, \sigma, \delta, p}^{\mu, \rho, \gamma}(z)
$$

$$
\begin{aligned}
& E_{\alpha, \beta, \nu, \sigma, \delta, p}^{\mu, \rho, \gamma, q}(z)=\frac{1}{\Gamma(\beta)}+\frac{(\mu)_{\rho}(\gamma)_{q}}{(\nu)_{\sigma}(\delta)_{p}} E_{\alpha+1, \beta, v+\sigma, \delta+p}^{\mu+\rho, \gamma+q}(z) \\
& E_{\alpha, \beta-\alpha, \nu, \sigma, \delta, p}^{\mu, \rho, \gamma, q}(z)-E_{\alpha, \beta-\alpha, \nu, \sigma, \delta, p}^{\mu, \rho, \gamma-1, q}(z) \\
& =\frac{q z(\mu)_{\rho}}{(v)_{\sigma}} \sum_{n=0}^{\infty} \frac{(n+1)(\mu+\rho)_{\rho n}(\gamma)_{q n+q-1}}{\Gamma(\alpha n+\beta)(\delta+p n)(v+\sigma)_{\sigma n}} \frac{z^{n}}{(\delta)_{p n}}
\end{aligned}
$$

In particular,

$$
\begin{gathered}
E_{\alpha, \beta, \delta}^{\gamma, q}(z)=\beta E_{\alpha, \beta+1, \delta}^{\gamma, q}(z)+\alpha z \frac{d}{d z} E_{\alpha, \beta+1, \delta}^{\gamma, q}(z), \\
E_{\alpha, \beta, \delta}^{\gamma, q}(z)=\frac{1}{\Gamma(\beta)}+\frac{(\gamma)_{q}}{\delta} z E_{\alpha, \alpha+\beta, \delta+1}^{\gamma+q, q}(z) \\
E_{\alpha, \beta-\alpha, \delta}^{\gamma, q}(z)-E_{\alpha, \beta-\alpha, \delta}^{\gamma-1, q}(z)=q z \sum_{n=0}^{\infty} \frac{(n+1)(\gamma)_{q n+q-1}}{\Gamma(\alpha n+\beta)(\delta+n)} \frac{z^{n}}{(\delta)_{n}}
\end{gathered}
$$

Proof.

$$
\begin{aligned}
& \beta E_{\alpha, \beta+1, v, \sigma, \delta, p}^{\mu, \rho, \gamma, q}(z)+\alpha z \frac{d}{d z} E_{\alpha, \beta+1, v, \sigma, \delta, p}^{\mu, \rho, \gamma, q}(z) \\
& =\beta E_{\alpha, \beta+1, v, \sigma, \delta, p}^{\mu, \rho, \gamma, q}(z)+\alpha z \frac{d}{d z} \sum_{n=0}^{\infty} \frac{(\mu)_{\rho n}(\gamma)_{q n}}{\Gamma(\alpha n+\beta+1)(v)_{\sigma n}} \frac{z^{n}}{(\delta)_{p n}} \\
& =\beta E_{\alpha, \beta+1, v, \sigma, \delta, p}^{\mu, \rho, \gamma, q}(z)+\sum_{n=0}^{\infty} \frac{\alpha n(\mu)_{\rho n}(\gamma)_{q n}}{\Gamma(\alpha n+\beta+1)(v)_{\sigma n}} \frac{z^{n}}{(\delta)_{p n}} \\
& =\beta E_{\alpha, \beta+1, v, \sigma, \delta, p}^{\mu, \rho, \gamma, q}(z)+\sum_{n=0}^{\infty} \frac{(\alpha n+\beta-\beta)(\mu)_{\rho n}(\gamma)_{q n}}{\Gamma(\alpha n+\beta+1)(v)_{\sigma n}} \frac{z^{n}}{(\delta)_{p n}}
\end{aligned}
$$

$$
=E_{\alpha, \beta, v, \sigma, \delta, p}^{\mu, \rho, \gamma, q}(z)
$$

which is (2.1.1).

The proof of (2.1.2) can easily be followed from the definition (1.9). Now

$$
\begin{aligned}
E_{\alpha, \beta-\alpha, v, \sigma, \delta, p}^{\mu, \rho, \gamma}, q & (z) E_{\alpha, \beta-\alpha, v, \sigma, \delta p}^{\mu, \rho, \gamma-1, q}(z) \\
= & \sum_{n=0}^{\infty} \frac{(\mu)_{\rho n}(\gamma)_{q n}}{\Gamma(\alpha(n-1)+\beta)(v)_{\sigma n}} \frac{z^{n}}{(\delta)_{p n}} \\
& -\sum_{n=0}^{\infty} \frac{(\mu)_{\rho n}(\gamma-1)_{q n}}{\Gamma(\alpha(n-1)+\beta)(v)_{\sigma n}} \frac{z^{n}}{(\delta)_{p n}} \\
= & q \sum_{n=1}^{\infty} \frac{n(\mu)_{\rho n}(\gamma)_{q n-1}}{\Gamma(\alpha(n-1)+\beta)} \frac{z^{n}}{(\delta)_{p n}} \\
= & \frac{(\mu)_{\rho} q z}{(\nu)_{\sigma}} \sum_{n=0}^{\infty} \frac{(n+1)(\mu+\rho)_{\rho n}(\gamma)_{q n+q-1}}{\Gamma(\alpha n+\beta)(v+\sigma)_{\sigma n}(\delta+p n)} \frac{z^{n}}{(\delta)_{p n}}
\end{aligned}
$$

which proves (2.1.3).

- Substituting $\mu=v, \rho=\sigma$ and $p=1$ in (2.1.1) immediately leads to (2.1.4).

- Substituting $\mu=v, \rho=\sigma$ and $p=1$ in (2.1.2) immediately leads to (2.1.5).

- Putting $\mu=v, \rho=\sigma$ and $p=1$ in (2.1.3) immediately leads to (2.1.6).

Theorem 2.2. If $\alpha, \beta, \gamma, \delta, \mu, v, \sigma, \rho, w \in \mathbb{C}, \operatorname{Re}(\alpha)>$ $0, \operatorname{Re}(\beta)>0, \operatorname{Re}(\gamma)>0, \operatorname{Re}(\delta)>0, \operatorname{Re}(\mu)>0, \operatorname{Re}(v)>$ $0, \operatorname{Re}(\rho)>0, \operatorname{Re}(\sigma)>0, \operatorname{Re}(\mathrm{w})>0 ;$ and $q \leq \operatorname{Re}(\alpha)+\mathrm{p}$ then for $m \in \mathbb{N}$

$$
\begin{aligned}
& \left(\frac{d}{d z}\right)^{m} E_{\alpha, \beta, \nu, \sigma, \delta, p}^{\mu, \rho, \gamma, q}(z) \\
& =\frac{(1)_{m}(\mu)_{\rho m}(\gamma)_{q m}}{(\nu)_{\sigma m}(\delta)_{p m}} \sum_{n=0}^{\infty} \\
& \quad \times \frac{(\mu+\rho m)_{\rho n}(\gamma+q m)_{q n}(1+m)_{n}}{\Gamma(\alpha n+\beta+\alpha)(v+\sigma m)_{\sigma n}(\delta+p m)_{p n}} \frac{z^{n}}{n !},
\end{aligned}
$$

$$
\begin{aligned}
\left(\frac{d}{d z}\right)^{m} & {\left[z^{\beta-1} E_{\alpha, \beta, v, \sigma, \delta, p}^{\mu, \rho, \gamma, q}\left(w z^{\alpha}\right)\right]=z^{\beta-m-1} E_{\alpha, \beta-m, v, \sigma, \delta, p}^{\mu, \rho, \gamma, q}\left(w z^{\alpha}\right), } \\
& \times \operatorname{Re}(\beta-m)>0,
\end{aligned}
$$

In particular,

$$
\left(\frac{d}{d z}\right)^{m} E_{\alpha, \beta, \delta}^{\gamma, q}(z)=\frac{(1)_{m}(\gamma)_{q m}}{(\delta)_{m}} \sum_{n=0}^{\infty} \frac{(\gamma+q m)_{q n}(1+m)_{n}}{\Gamma\left(\alpha n+\beta+\alpha(\delta+m)_{n}\right.} \frac{z^{n}}{n !},
$$




$$
\begin{aligned}
& \left(\frac{d}{d z}\right)^{m}\left[z^{\beta-1} E_{\alpha, \beta, \delta}^{\gamma, q}\left(w z^{\alpha}\right)\right]=z^{\beta-m-1} E_{\alpha, \beta-m, p}^{\gamma, q}\left(w z^{\alpha}\right), \\
& \times \operatorname{Re}(\beta-m)>0
\end{aligned}
$$

Proof. From (1.9),

$$
\begin{aligned}
\left(\frac{d}{d z}\right)^{m} & \sum_{n=0}^{\infty} \frac{(\mu)_{\rho n}(\gamma)_{q n}}{\Gamma(\alpha n+\beta)(\nu)_{\sigma n}} \frac{z^{n}}{(\delta)_{p n}} \\
= & \sum_{n=m}^{\infty} \frac{(\mu)_{\rho n}(\gamma)_{q n} n !}{\Gamma(\alpha n+\beta)(\nu)_{\sigma n}(\delta)_{p n}} \frac{z^{n-m}}{(n-m) !} \\
= & \frac{(1)_{m}(\mu)_{\rho m}(\gamma)_{q m}}{(\nu)_{\sigma m}(\delta)_{p m}} \sum_{n=0}^{\infty} \\
& \times \frac{(\mu+\rho)_{\rho m}(\gamma+q m)_{q n}(1+m)_{n}}{\Gamma(\alpha n+\alpha m+\beta)(\nu+\sigma)_{\sigma m}(\delta+p m)_{p n}} \frac{z^{n}}{n !}
\end{aligned}
$$

which is the proof of (2.2.1).

Again using (1.9) and term by term differentiation under the sign summation(which is possible in accordance with the uniform convergence of the series (1.9) in any compact set $\mathbb{C}$ ), we have

$$
\begin{aligned}
\left(\frac{d}{d z}\right)^{m} & {\left[z^{\beta-1} E_{\alpha, \beta, v, \sigma, \delta, p}^{\mu, \rho, \gamma, q}\left(w z^{\alpha}\right)\right] } \\
& =\left(\frac{d}{d z}\right)^{m} \sum_{n=0}^{\infty} \frac{(\mu)_{\rho n}(\gamma)_{q n} w^{n}}{\Gamma(\alpha n+\beta)_{\sigma n}} \frac{z^{\alpha n+\beta-1}}{(\delta)_{p n}} \\
& =\sum_{n=0}^{\infty} \frac{(\mu)_{\rho n}(\gamma)_{q n}(\alpha n+\beta-1) ! w^{n}}{\Gamma(\alpha n+\beta)(\alpha n+\beta-1-m) !(v)_{\sigma n}} \frac{z^{\alpha n+\beta-m-1}}{(\delta)_{p n}} \\
& =z^{\beta-m-1} E_{\alpha, \beta-m, v, \sigma, \delta, p}^{\mu, \rho, \gamma, q}\left(w z^{\alpha}\right)
\end{aligned}
$$

which is the proof of (2.2.2).

- Setting $\mu=\rho, v=\sigma$, in (2.2.1), we get (2.2.3).

- Setting $\mu=\rho, v=\sigma$, in (2.2.2), we get (2.2.4).

Theorem 2.3. If $\alpha=\frac{m}{r}$, with $m, r \in \mathbb{N}$ relatively prime; $\beta, \gamma, \delta, \mu, \nu, \rho, \sigma \in \mathbb{C}$ and $\mathrm{q}<\operatorname{Re}(\alpha+\mathrm{p})$, then

$$
\begin{aligned}
& \frac{d^{m}}{d z^{m}} E_{\frac{m}{r}, \beta, \nu, \sigma, \delta, p}^{\mu, \rho, \gamma, q}\left(z^{\frac{p}{r}}\right)=\frac{\Gamma(\nu)}{\Gamma(\mu)} \sum_{n=1}^{\infty} \\
& \quad \times \frac{\Gamma\left(\frac{n m}{r}+1\right) \Gamma(\mu+\rho n)(\gamma)_{q n}}{\Gamma\left(\frac{n m}{r}+\beta\right) \Gamma\left(\frac{n m}{r}-m+1\right) \Gamma(v+\sigma n)} \frac{z^{\left(\frac{n}{r}-1\right) m}}{(\delta)_{p n}}
\end{aligned}
$$

$$
\begin{aligned}
& \frac{d^{m}}{d z^{m}} E_{\frac{m}{r}, \beta, \delta}^{\gamma, q}\left(z^{\frac{m}{r}}\right)=\Gamma(\delta) \sum_{n=1}^{\infty} \\
& \quad \times \frac{(\gamma)_{q n} \Gamma(n+1) \Gamma\left(\frac{n m}{r}+1\right)}{\Gamma(\delta+n) \Gamma\left(\frac{n m}{r}+\beta\right) \Gamma\left(\frac{n p}{r}-m+1\right)} \frac{z^{\left(\frac{n}{r}-1\right) m}}{n !}
\end{aligned}
$$

Proof.

$$
\begin{aligned}
\frac{d^{m}}{d z^{m}} & E_{\frac{m}{r}, \beta, v, \sigma, \delta, p}^{\mu, \rho, \gamma, q}\left(z^{\frac{p}{r}}\right) \\
& =\frac{d^{m}}{d z^{m}} \sum_{n=0}^{\infty} \frac{(\mu)_{\rho n}(\gamma)_{q n}}{\Gamma\left(\frac{n m}{r}+\beta\right)(v)_{\sigma n}} \frac{z^{\left(\frac{m}{r}\right) n}}{(\delta)_{p n}} \\
& =\sum_{n=1}^{\infty} \frac{(\mu)_{\rho n}(\gamma)_{q n}\left(\frac{n m}{r}\right) !}{\Gamma\left(\frac{n m}{r}+\beta\right)(v)_{\sigma n}\left(\frac{n m}{r}-m\right) !} \frac{z^{\left(\frac{n}{r}-1\right) m}}{(\delta)_{p n}} \\
& =\frac{\Gamma(v)}{\Gamma(\mu)} \sum_{n=1}^{\infty} \frac{\Gamma\left(\frac{n m}{r}+1\right) \Gamma(\mu+\rho n)(\gamma)_{q n}}{\Gamma\left(\frac{n m}{r}+\beta\right) \Gamma\left(\frac{n m}{r}-m+1\right) \Gamma(v+\sigma n)} \frac{z^{\left(\frac{n}{r}-1\right) m}}{(\delta)_{p n}}
\end{aligned}
$$

which proves (2.3.1).

Corollary 2.3. For $\mu=v, \rho=\sigma, \delta=p=1$, (2.3.1) reduces to the known result of Shukla and Prajapati Shukla and Prajapati (2007) (2.3.1).

Remark 2.3. Setting $\mu=v, \rho=\sigma$ and $p=1$ in (2.3.1), we get (2.3.2).

Special Properties: Setting putting $\mu=v, \rho=\sigma$ and $p=q=\delta=1$ in (2.3.1), we have

$$
\frac{d^{m}}{d z^{m}} E_{\frac{m}{r}, \beta}^{\gamma}\left(z^{\frac{m}{r}}\right)=\sum_{n=1}^{\infty} \frac{(\gamma)_{n} \Gamma\left(\frac{n p}{r}+1\right)}{\Gamma\left(\frac{n m}{r}+\beta\right) \Gamma\left(\frac{n p}{r}-m+1\right)} \frac{z^{\left(\frac{n}{r}-1\right) m}}{n !}
$$

For $\beta=\gamma=\delta=q=1$ in (2.3.2), we have

$$
\frac{d^{m}}{d z^{m}} E_{\frac{m}{r}}\left(z^{\frac{m}{r}}\right)=\sum_{n=1}^{\infty} \frac{z^{\left(\frac{n}{r}-1\right) m}}{\Gamma\left(\frac{n m}{r}-m+1\right)}
$$

Theorem 2.4. If $\alpha, \beta, \mu, \rho, \nu, \gamma, \lambda, \sigma, \delta, \eta \in \mathbb{C} ; \operatorname{Re}(\alpha)>$ $0, \operatorname{Re}(\beta)>0, \operatorname{Re}(\gamma)>0, \operatorname{Re}(\mu)>0, \operatorname{Re}(\rho)>0, \operatorname{Re}(v)>$ $0, \operatorname{Re}(\delta)>0, \operatorname{Re}(\sigma)>0, \operatorname{Re}(\lambda)>0$ and $\mathrm{q}<\operatorname{Re}(\alpha)+\mathrm{p}$ then

$$
\begin{gathered}
\frac{1}{\Gamma(\eta)} \int_{0}^{1} u^{\beta-1}(1-u)^{\eta-1} E_{\alpha, \beta, v, \sigma, \delta, p}^{\mu, \rho, \gamma, q}\left(z u^{\alpha}\right) d u \\
\quad=E_{\alpha, \beta+\eta, v, \sigma, \delta, p}^{\mu, \rho, \gamma, q}(z) d u
\end{gathered}
$$




$$
\begin{aligned}
& \frac{1}{\Gamma(\eta)} \int_{t}^{x}(x-s)^{\eta-1}(s-t)^{\beta-1} E_{\alpha, \beta, \nu, \sigma, \delta, p}^{\mu, \rho, \gamma}\left[\lambda(s-t)^{\alpha}\right] d u \\
& \quad=(x-t)^{\eta+\beta-1} E_{\alpha, \beta+\eta, \delta, p}^{\mu, \rho, \gamma, q}(z), \\
& \int_{0}^{z} t^{\beta-1} E_{\alpha, \beta, \mu, \rho, \delta, p}^{\mu, \rho, \gamma, q}\left(w t^{\alpha}\right) d t=z^{\beta} E_{\alpha, \beta+1, \nu, \sigma, \delta, p}^{\mu, \rho, \gamma, q}\left(w z^{\alpha}\right)
\end{aligned}
$$

In particular,

$$
\frac{1}{\Gamma(\eta)} \int_{0}^{1} u^{\beta-1}(1-u)^{\sigma-1} E_{\alpha, \beta, \delta}^{\gamma, q}\left(z u^{\alpha}\right) d u=E_{\alpha, \beta+\eta, \delta}^{\gamma, q}(z) d u
$$

$$
\begin{aligned}
& \frac{1}{\Gamma(\eta)} \int_{t}^{x}(x-s)^{\eta-1}(s-t)^{\beta-1} E_{\alpha, \beta, \delta}^{\gamma, q}\left[\lambda(s-t)^{\alpha}\right] d u \\
& \quad=(x-t)^{\eta+\beta-1} E_{\alpha, \beta+\eta, \delta}^{\gamma, q}(z), \\
& \int_{0}^{z} t^{\beta-1} E_{\alpha, \beta, \delta}^{\gamma, q}\left(w t^{\alpha}\right) d t=z^{\beta} E_{\alpha, \beta+1, \delta}^{\gamma, q}\left(w z^{\alpha}\right), \\
& \frac{1}{\Gamma(\eta)} \int_{0}^{1} u^{\beta-1}(1-u)^{\sigma-1} E_{\alpha, \beta}^{\gamma}\left(z u^{\alpha}\right) d u=E_{\alpha, \beta+\eta}^{\gamma}(z) d u
\end{aligned}
$$

$$
\int_{0}^{z} t^{\beta-1} E_{\alpha, \beta}\left(w t^{\alpha}\right) d t=z^{\beta} E_{\alpha, \beta+1}\left(w z^{\alpha}\right)
$$

\section{Proof.}

$$
\begin{aligned}
\frac{1}{\Gamma(\eta)} & \int_{0}^{1} u^{\beta-1}(1-u)^{\eta-1} E_{\alpha, \beta, v, \sigma, \delta, p}^{\mu, \rho, \gamma, q}\left(z u^{\alpha}\right) d u \\
= & \frac{1}{\Gamma(\eta)} \sum_{n=0}^{\infty} \frac{(\mu)_{\rho n}(\gamma)_{q n} z^{n}}{\Gamma(\alpha n+\beta)(\nu)_{\sigma n}(\delta)_{p n}} \int_{0}^{1} \\
& \times u^{\alpha n+\beta-1}(1-u)^{\eta-1} d u \\
= & \frac{1}{\Gamma(\eta)} \sum_{n=0}^{\infty} \frac{(\mu)_{\rho n}(\gamma)_{q n} z^{n}}{\Gamma(\alpha n+\beta)(\nu)_{\sigma n}(\delta)_{p n}} B(\alpha n+\beta, \eta) \\
= & E_{\alpha, \beta+\eta, \nu, \sigma, \delta, p}^{\mu, \rho, q}(z)
\end{aligned}
$$

which proves (2.4.1).

Now change the variable from $s$ to $u=\frac{s-t}{x-t}$. Then the L.H.S. of (2.4.2) becomes

$$
\begin{aligned}
\frac{1}{\Gamma(\eta)} \int_{0}^{1} & (x-t)^{\eta+\beta-1}(1-u)^{\eta-1} u^{\beta-1} \\
& \times \sum_{n=0}^{\infty} \frac{(\mu)_{\rho n}(\gamma)_{q n} z^{n}}{\Gamma(\alpha n+\beta)(\nu)_{\sigma n}(\delta)_{p n}}(\lambda)^{n}(x-t)^{\alpha n} u^{\alpha n} d u
\end{aligned}
$$

$$
\begin{aligned}
= & \frac{(x-t)^{\eta+\beta-1}}{\Gamma(\eta)} \sum_{n=0}^{\infty} \frac{(\mu)_{\rho n}(\gamma)_{q n}\left[\lambda(x-t)^{\alpha}\right]^{n}}{\Gamma(\alpha n+\beta)(\nu)_{\sigma n}(\delta)_{p n}} \int_{0}^{1} \\
& \times u^{\alpha n+\beta-1}(1-u)^{\eta-1} d u \\
= & \frac{(x-t)^{\eta+\beta-1}}{\Gamma(\eta)} \sum_{n=0}^{\infty} \frac{(\gamma)_{q n}\left[\lambda(x-t)^{\alpha}\right]^{n}}{\Gamma(\alpha n+\beta)(\delta)_{p n}} B(\alpha n+\beta, \eta) \\
= & (x-t)^{\eta+\beta-1} E_{\alpha, \beta+\eta, \nu, \sigma, \delta, p}^{\mu, \rho, q}\left[\lambda(x-t)^{\alpha}\right]^{n}
\end{aligned}
$$

which proves (2.4.2).

Now

$$
\begin{aligned}
& \int_{0}^{z} t^{\beta-1} E_{\alpha, \beta, \nu, \sigma, \sigma, \delta, p}^{\mu, \rho, \gamma, q}\left(w t^{\alpha}\right) d t \\
& \quad=\int_{0}^{z} t^{\beta-1} \sum_{n=0}^{\infty} \frac{(\mu)_{\rho n}(\gamma)_{q n} w^{n} t^{\alpha n}}{\Gamma(\alpha n+\beta)(\nu)_{\sigma n}(\delta)_{p n}} d t \\
& \quad=\sum_{n=0}^{\infty} \frac{(\mu)_{\rho n}(\gamma)_{q n} w^{n}}{\Gamma(\alpha n+\beta)(\nu)_{\sigma n}(\delta)_{p n}} \int_{0}^{z} t^{\alpha n+\beta-1} d t \\
& =z^{\beta} \sum_{n=0}^{\infty} \frac{(\mu)_{\rho n}(\gamma)_{q n}\left(w z^{\alpha}\right)^{n}}{\Gamma(\alpha n+\beta+1)(\nu)_{\sigma n}(\delta)_{p n}} \\
& =z^{\beta} E_{\alpha, \beta+1, \nu, \sigma, \delta, p}^{\mu, \rho, \gamma, q}\left(w z^{\alpha}\right)
\end{aligned}
$$

which proves (2.4.3).

Putting $q=\delta=1$ and $\gamma=q=\delta=1$ in (2.4.1) and (2.4.3) yields (2.4.4) and (2.4.5) respectively.

\section{Generalized hypergeometric function}

representation of $E_{\alpha, \beta, v, \sigma, \delta, p}^{\mu, \rho, \gamma, q}$

Using (1.9) with $\alpha=k, \rho=l, \sigma=m \in \mathbb{N}$ and $q \in \mathbb{N}$, we have

$$
\begin{aligned}
E_{\alpha, \beta, \nu, \sigma, \delta, p}^{\mu, \rho, \gamma, q}(z) & =\sum_{n=0}^{\infty} \frac{(\mu)_{\ln }(\gamma)_{q n}}{\Gamma(k n+\beta)(\nu)_{m n}(\delta)_{p n}} z^{n} \\
= & \frac{1}{\Gamma(\beta)} \sum_{n=0}^{\infty} \frac{(\mu)_{l n}(\gamma)_{q n}(1)_{n}}{(\beta)_{k n}(\nu)_{m n}(\delta)_{p n}} \frac{z^{n}}{n !} \\
= & \frac{1}{\Gamma(\beta)} \sum_{n=0}^{\infty} \frac{\prod_{i=1}^{l}\left(\frac{\mu+i-1}{l}\right)_{n} \prod_{j=1}^{q}\left(\frac{\gamma+j-1}{q}\right)_{n}(1)_{n}}{\prod_{r=1}^{k}\left(\frac{\beta+r-1}{k}\right)_{n} \prod_{s=1}^{p}\left(\frac{\delta+s-1}{p}\right)_{n} \prod_{t=1}^{m}\left(\frac{v+t-1}{m}\right)_{n} n !} \\
& \times\left(\frac{l^{l} q^{q} z}{m^{m} p^{p} k^{k}}\right)^{n} \\
= & \frac{1}{\Gamma(\beta)} q+l+1 F_{k+p+m}[\Delta(l ; \mu), \Delta(q ; \gamma),(1,1) ; \Delta(k, \beta), \\
& \left.\Delta(p ; \delta), \Delta(m ; v) ; \frac{l^{l} q^{q} z}{m^{m} p^{p} k^{k}}\right]
\end{aligned}
$$

where $\Delta(l ; \mu)$ is a $l$-tupple $\frac{\mu}{l}, \frac{\mu+1}{l}, \ldots \frac{\mu+l-1}{l} ; \Delta(q ; \gamma)$ is a $q$-tupple $\frac{\gamma}{q}, \frac{\gamma+1}{q}, \ldots \frac{\gamma+q-1}{q} ; \Delta(k, \beta)$ is a $k$-tupple $\frac{\beta}{k}, \frac{\beta+1}{k}, \ldots, \frac{\beta+k-1}{k}$ and so on, which is the required hypergeometric representation. 
Convergence criterion of generalized Mittag-leffler function ${ }_{q+l+1} F_{k+p+m}$ :

(i) If $q+l+1 \leq k+p+m$, the function ${ }_{q+l+1} F_{k+p+m}$ converges for all finite $\mathrm{z}$.

(ii) If $q+l+1=k+p+m+1$, the function ${ }_{q+l+1} F_{k+p+m}$ converges for $|z|<1$ and diverges for $|z|>1$

(iii) If $q+l+1>k+p+m+1$, the function $q+1+1 F_{k+p+m+1}$ is divergent for $|z| \neq 0$

(iv) If $q+l+1=k+p+m+1$, the function ${ }_{q+l+1} F_{k+p+m+1}$ is absolutely convergent on the circle for $|z|=1$, if

$$
\begin{gathered}
\operatorname{Re}\left(\sum_{r=1}^{k} \sum_{s=1}^{p} \sum_{t=1}^{m} \frac{\beta+r-1}{k} \cdot \frac{\delta+s-1}{p} \frac{\nu+t-1}{m}\right. \\
\left.-\sum_{i=1}^{l} \sum_{j=1}^{q} \frac{\mu+i-1}{l} \frac{\gamma+j-1}{q}\right)>0
\end{gathered}
$$

\section{Integral transforms of $E_{\alpha, \beta, v, \sigma, \delta, p}^{\mu, \rho, \gamma, q}(z)$}

In this section we discuss some useful integral transforms like Euler transform, laplace transform and Whittaker transform of $E_{\alpha, \beta, \nu, \sigma, \delta, p}^{\mu, \rho, \gamma, q}(z)$.

Theorem 4.1. Mellin-Barnes integral representation of $E_{\alpha, \beta, v, \sigma, \delta, p}^{\mu, \rho, q)}(z)$

Let (1.9) and (1.10) be satified and $\alpha \in R_{+}=(0 \infty)$; $\beta, \nu, \sigma, \mu, \rho, \gamma, \delta \in \mathbb{C}$ and $q<\operatorname{Re}(\alpha)+p$. Then the function $E_{\alpha, \beta, \delta}^{\gamma, q}(z)$ is represented by Mellin-Barnes integral as:

$$
\begin{aligned}
& E_{\alpha, \beta, v, \sigma, \delta, p}^{\mu, \rho, \gamma, q}(z)=\frac{\Gamma(\delta) \Gamma(v)}{2 \pi i \Gamma(\gamma) \Gamma(\mu)} \int_{L} \\
& \quad \times \frac{\Gamma(s) \Gamma(1-s) \Gamma(\mu-\rho s) \Gamma(\gamma-q s)}{\Gamma(\beta-\alpha s) \Gamma(\delta-p s) \Gamma(v-\rho s)}(-z)^{-s} d s
\end{aligned}
$$

where $|\arg (z)|<1$; the contour of integration beginning at $-i \infty$ and ending at $+i \infty$, and indented to separate the poles of the integrand at $s=-n, \forall n \in \mathbb{N}_{0}$ (to the left) from those at $s=\frac{\gamma+n}{q}, n \in \mathbb{N}_{0}$ (to the right).

Proof. We shall evaluate the integral on R.H.S. of (4.1.1) as the sum of the residues at the poles $s=0,-1,-2, \ldots$, we have

$$
\begin{aligned}
\frac{1}{2 \pi i} & \int_{L} \frac{\Gamma(s) \Gamma(1-s) \Gamma(\mu-\rho s) \Gamma(\gamma-q s)}{\Gamma(\beta-\alpha s) \Gamma(v-\sigma s) \Gamma(\delta-p s)}(-z)^{-s} d s \\
= & \sum_{n=0}^{\infty} \operatorname{Res}_{s=-n}\left[\frac{\Gamma(s) \Gamma(1-s) \Gamma(\mu-\rho s) \Gamma(\gamma-q s)}{\Gamma(\beta-\alpha s) \Gamma(\delta-p s) \Gamma(v-\sigma s)}(-z)^{-s}\right] \\
= & \sum_{n=0}^{\infty} \operatorname{Lim}_{s \rightarrow-n} \frac{\pi(s+n)}{\sin \pi s} \cdot \frac{\Gamma(\mu-\rho s) \Gamma(\gamma-q s)}{\Gamma(\beta-\alpha s) \Gamma(\delta-p s) \Gamma(v-\sigma s)} \\
& \times(-z)^{-s}
\end{aligned}
$$

$$
\begin{aligned}
& =\sum_{n=0}^{\infty} \frac{(-1)^{n} \Gamma(\mu+\rho n) \Gamma(\gamma+q n)}{\Gamma(\beta+\alpha n) \Gamma(\delta+p n) \Gamma(v+\sigma n)}(-z)^{n} \\
& =\frac{\Gamma(\gamma) \Gamma(\mu)}{\Gamma(\delta) \Gamma(v)} \sum_{n=0}^{\infty} \frac{(\mu)_{\rho n}(\gamma)_{q n}}{\Gamma(\alpha n+\beta)(\delta)_{p n}(v)_{\sigma n}} z^{n} \\
& =\frac{\Gamma(\gamma) \Gamma(\mu)}{\Gamma(\delta) \Gamma(v)} E_{\alpha, \beta, \nu, \sigma, \delta, p}^{\mu, \rho, \gamma, q}(z)
\end{aligned}
$$

which completes the proof.

Remark 4.1. Setting $\mu=\rho, v=\sigma$ and $p=1$, we get the Melin Barne's integral of the function $E_{\alpha, \beta, \delta}^{\gamma, q}$.

Theorem 4.2. (Mellin transform) of $E_{\alpha, \beta, v, \sigma, \delta, p}^{\mu, \rho, q, q}(z)$

$$
\begin{aligned}
& M\left\{E_{\alpha, \beta, v, \sigma, \delta, p}^{\mu, \rho, \gamma, q}(-w z) ; s\right\}=\frac{\Gamma(\delta) \Gamma(v)}{2 \pi i \Gamma(\gamma) \Gamma(\mu)} \int_{L} \\
& \quad \times \frac{\Gamma(s) \Gamma(1-s) \Gamma(\mu-\rho s) \Gamma(1-q s)}{\Gamma(\gamma) \Gamma(\beta-\alpha s) \Gamma(v-\sigma s) \Gamma(\delta-p s)}(w z)^{-s} d s,
\end{aligned}
$$

Proof. From Theorem 4.1, we have

$$
\begin{aligned}
& E_{\alpha, \beta, \nu, \sigma \sigma, \delta, p}^{\mu, \rho, \gamma, q}(-w z) \\
& =\frac{\Gamma(\delta) \Gamma(v)}{2 \pi i \Gamma(\gamma) \Gamma(\mu)} \int_{L} \frac{\Gamma(s) \Gamma(1-s) \Gamma(\mu-\rho s) \Gamma(\gamma-q s)}{\Gamma(\beta-\alpha s) \Gamma(v-\sigma s) \Gamma(\delta-p s)} \\
& \quad \times(w z)^{-s} d s \\
& =\frac{\Gamma(\delta) \Gamma(v)}{2 \pi i \Gamma(\gamma) \Gamma(\mu)} \int_{L} f^{*}(s)(z)^{-s} d s
\end{aligned}
$$

where

$$
f^{*}(s)=\frac{\Gamma(s) \Gamma(1-s) \Gamma(\mu-\rho s) \Gamma(\gamma-q s)}{\Gamma(\beta-\alpha s) \Gamma(v-\sigma s) \Gamma(\delta-p s)}(w)^{-s}
$$

is in the form of inverse Mellin-Transform (1.15). So applying the Mellin-transform (1.14) yields directly the required result.

Theorem 4.3. (Euler(Beta)transform) of $E_{\alpha, \beta, v, \sigma, \delta, p}^{\mu, \rho, \gamma, q}(z)$

$$
\begin{aligned}
\int_{0}^{1} z^{a-1}(1-z)^{b-1} E_{\alpha, \beta, \nu, \sigma, \delta, p}^{\mu, \rho, \gamma, q}\left(x z^{\eta}\right) d z \\
\quad=\frac{\Gamma(b) \Gamma(v) \Gamma(\delta)}{\Gamma(\mu) \Gamma(\gamma)}{ }_{4} \Psi_{4}\left[\begin{array}{l}
(\mu, \rho)(\gamma, q),(a, \eta),(1,1) \quad ; \quad x \\
(\beta, \alpha),(a+b, \eta),(\nu, \sigma),(\delta, p) ;
\end{array}\right]
\end{aligned}
$$

Proof.

$$
\begin{aligned}
& \int_{0}^{1} z^{a-1}(1-z)^{b-1} E_{\alpha, \beta, \nu, \sigma, \delta, p}^{\mu, \rho, \gamma, q}\left(x z^{\eta}\right) d z \\
& =\int_{0}^{1} z^{a-1}(1-z)^{b-1} \sum_{n=0}^{\infty} \frac{(\mu)_{\rho n}(\gamma)_{q n}\left(x z^{\eta}\right)^{n}}{\Gamma(\alpha n+\beta)(\nu)_{\sigma n}(\delta)_{p n}}
\end{aligned}
$$




$$
\begin{aligned}
= & \sum_{n=0}^{\infty} \frac{(\mu)_{\rho n}(\gamma)_{q n} x^{n}}{\Gamma(\alpha n+\beta)(v)_{\sigma n}(\delta)_{p n}} \int_{0}^{1} z^{\eta n+a-1}(1-z)^{b-1} d z \\
= & \sum_{n=0}^{\infty} \frac{(\mu)_{\rho n}(\gamma)_{q n} x^{n}}{\Gamma(\alpha n+\beta)(v)_{\sigma n}(\delta)_{p n}} B(\eta n+a, b) \\
= & \frac{\Gamma(b) \Gamma(v) \Gamma(\delta)}{\Gamma(\mu) \Gamma(\gamma)} \sum_{n=0}^{\infty} \\
& \times \frac{\Gamma(\mu+\rho n) \Gamma(q n+\gamma) \Gamma(a+\eta n) \Gamma(n+1)}{\Gamma(\alpha n+\beta) \Gamma(\eta n+a+b) \Gamma(v+\sigma n) \Gamma(p n+\delta)} \frac{x^{n}}{n !},
\end{aligned}
$$

from which the result follows.

\section{Corollary 4.3.}

$$
\begin{aligned}
& \int_{0}^{1} z^{a-1}(1-z)^{b-1} E_{\alpha, \beta, \delta}^{\gamma, q}\left(x z^{\eta}\right) d z \\
& \quad=\frac{\Gamma(b) \Gamma(\delta)}{\Gamma(\gamma)}{ }_{3} \Psi_{3}\left[\begin{array}{ll}
(\gamma, q),(a, \eta),(1,1) & ; \\
(\beta, \alpha),(a+b, \eta),(\delta, 1) ;
\end{array}\right]
\end{aligned}
$$

Special properties: (i) For $q=1,(4.3 .2)$ reduces to Tariq O Salim (2009)(4.1).

$$
\begin{aligned}
& \int_{0}^{1} z^{a-1}(1-z)^{b-1} E_{\alpha, \beta}^{\gamma, \delta}\left(x z^{\sigma}\right) d z \\
& =\frac{\Gamma(b) \Gamma(\delta)}{\Gamma(\gamma)}{ }_{3} \Psi_{3}\left[\begin{array}{ll}
(\gamma, 1),(a, \sigma),(1,1) & ; \\
(\beta, \alpha),(\delta, 1)(a+b, \sigma) ; & x
\end{array}\right],
\end{aligned}
$$

(ii) For $\delta=q=1$ in (4.3.2), we have

$$
\begin{aligned}
& \int_{0}^{1} z^{a-1}(1-z)^{b-1} E_{\alpha, \beta}^{\gamma}\left(x z^{\sigma}\right) d z \\
& \quad=\frac{\Gamma(b)}{\Gamma(\gamma)}{ }_{2} \Psi_{2}\left[\begin{array}{ll}
(\gamma, 1),(a, \sigma), & ; \\
(\beta, \alpha),(a+b, \sigma) ;
\end{array}\right],
\end{aligned}
$$

If $a=\beta, \alpha=\sigma$, then (4.3.2) reduces to

$$
\int_{0}^{1} z^{\beta-1}(1-z)^{b-1} E_{\sigma, \beta, \delta}^{\gamma, q}\left(x z^{\sigma}\right) d z=\frac{\Gamma(b)}{\Gamma(\gamma)} E_{\sigma, \beta+b, \delta}^{\gamma, q}(x),
$$

Putting $\alpha=\beta=\gamma=\delta=q=1$ in (4.3.2), we have

$$
\begin{aligned}
& \int_{0}^{1} z^{a-1}(1-z)^{b-1} \exp \left(x z^{\sigma}\right) d z \\
& =\Gamma(b)_{1} \Psi_{1}\left[\begin{array}{ll}
(a, \sigma), & ; \\
(a+b, \sigma) ; & x
\end{array}\right]
\end{aligned}
$$

\section{Theorem 4.4. (Laplace transform)}

$$
\begin{aligned}
\int_{0}^{\infty} z^{a-1} e^{-s z} E_{\alpha, \beta, \nu, \sigma, \delta, p}^{\mu, \rho, \gamma, q}\left(x z^{\eta}\right) d z \\
=\frac{\Gamma(\nu) \Gamma(\delta)}{\Gamma(\mu) \Gamma(\gamma)} s^{-a_{3} \Psi_{3}}\left[\begin{array}{l}
(\mu, \rho)(\gamma, q),(1,1) ; \\
(\beta, \alpha),(\nu, \sigma)(\delta, p) ;
\end{array}\right],
\end{aligned}
$$

\section{Proof.}

$\int_{0}^{\infty} z^{a-1} e^{-s z} E_{\alpha, \beta, v, \sigma, \delta, p}^{\mu, \rho, q, q}\left(x z^{\eta}\right) d z$

$$
\begin{aligned}
& =\int_{0}^{\infty} z^{a-1} e^{-s z} \sum_{n=0}^{\infty} \frac{(\mu)_{\rho n}(\gamma)_{q n}\left(x z^{\sigma}\right)^{n}}{\Gamma(\alpha n+\beta)(v)_{\sigma n}(\delta)_{p n}} d z \\
& =\frac{\Gamma(v) \Gamma(\delta) s^{-a}}{\Gamma(\mu) \Gamma(\gamma)} \sum_{n=0}^{\infty} \frac{\Gamma(\mu+\rho n) \Gamma(\gamma+q n) \Gamma(1+n)}{\Gamma(\beta+\alpha n) \Gamma(v+\sigma n) \Gamma(\delta+p n)} \frac{\left(\frac{x}{\sigma^{\sigma}}\right)^{n}}{n !},
\end{aligned}
$$

from which the result follows.

\section{Corollary 4.4.}

$$
\begin{aligned}
& \int_{0}^{\infty} z^{a-1} e^{-s z} E_{\alpha, \beta, \delta}^{\gamma, q}\left(x z^{\eta}\right) d z \\
& =\frac{\Gamma(\delta)}{\Gamma(\gamma)} s_{2}^{-a} \Psi_{2}\left[\begin{array}{l}
(\gamma, q),(1,1) ; \\
(\beta, \alpha),(\delta, 1) ;
\end{array}\right],
\end{aligned}
$$

Remark 4.4. For $q=1,(4.4 .2)$ reduces to Tariq $\mathrm{O}$ Salim (2009)(4.2).

\section{Theorem 4.5. (Whittaker transform)}

$$
\begin{gathered}
\int_{0}^{\infty} e^{\frac{-\phi t}{2}} t^{\xi-1} W_{\lambda, \psi}(\phi t) E_{\alpha, \beta, \nu, \sigma, \delta, p}^{\mu, \rho, \gamma}\left(\omega t^{\eta}\right) d t=\frac{\Gamma(\nu) \Gamma(\delta)}{\Gamma(\mu) \Gamma(\gamma)} \phi^{-\xi} \Psi_{4} \\
\times\left[\begin{array}{lr}
(\mu, \rho)(\gamma, q),\left(\frac{1}{2}+\psi+\xi, \eta\right),\left(\frac{1}{2}-\psi+\xi, \eta\right),(1,1) & ; \\
(\beta, \alpha),(\nu, \sigma),(1-\lambda+\xi, \eta),(\delta, p) & \frac{\omega}{\phi^{\eta}}
\end{array}\right]
\end{gathered}
$$

Proof. Substituting $\phi t=v$ in L.H.S. of Theorem 4.5, we have

$$
\begin{aligned}
\int_{0}^{\infty}\left(\frac{v}{\phi}\right)^{\xi-1} e^{\frac{-v}{2}} W_{\lambda, \psi}(v) \sum_{n=0}^{\infty} \frac{(\mu)_{\rho n}(\gamma)_{q n}(\omega)^{n}}{\Gamma(\alpha n+\beta)(\delta)_{n}}\left(\frac{v}{\phi}\right)^{n \eta} \frac{1}{\phi} d v \\
=\frac{\phi^{-\xi} \Gamma(v) \Gamma(\delta)}{\Gamma(\mu) \Gamma(\gamma)} \sum_{n=0}^{\infty} \frac{\Gamma(\mu+\rho n) \Gamma(\gamma+q n)}{\Gamma(\alpha n+\beta) \Gamma(v+\sigma n) \Gamma(\delta+p n} \\
\quad \times\left(\frac{\omega}{\phi^{\eta}}\right)^{n} \int_{0}^{\infty}(v)^{\eta n+\xi-1} e^{\frac{-v}{2}} W_{\lambda, \psi}(v) d v \\
=\frac{\phi^{-\xi} \Gamma(v) \Gamma(\delta)}{\Gamma(\mu) \Gamma(\gamma)} \sum_{n=0}^{\infty} \frac{\Gamma(\mu+\rho n) \Gamma(\gamma+q n)}{\Gamma(\alpha n+\beta) \Gamma(v+\sigma n) \Gamma(\delta+p n)} \\
\quad \times\left(\frac{\omega}{\phi^{\eta}}\right)^{n} \frac{\Gamma\left(\frac{1}{2}+\psi+\xi+n \eta\right) \Gamma\left(\frac{1}{2}-\psi+\xi+n \eta\right)}{\Gamma(1-\lambda+\xi+n \eta)}
\end{aligned}
$$




$$
\begin{aligned}
= & \frac{\phi^{-\xi} \Gamma(v) \Gamma(\delta)}{\Gamma(\mu) \Gamma(\gamma)} \sum_{n=0}^{\infty} \\
& \times \frac{\Gamma(\mu+\rho n) \Gamma(\gamma+q n) \Gamma\left(\frac{1}{2} \pm \psi+\xi+n \eta\right) \Gamma(n+1)}{\Gamma(1-\lambda+\xi+n \eta)} \\
& \times \frac{\left(\frac{\omega}{\phi^{\eta}}\right)^{n}}{n !},
\end{aligned}
$$

from which the result follows.

\section{Corollary 4.5.}

$$
\begin{aligned}
& \int_{0}^{\infty} \mathrm{e}^{\frac{-\phi t}{2} t^{\xi-1} W_{\lambda, \psi}(\phi t) E_{\alpha, \beta, \delta}^{\gamma, q}\left(\omega t^{\eta}\right) d t=\frac{\Gamma(\delta)}{\Gamma(\gamma)} \phi^{-\xi}{ }_{4} \Psi_{3}} \\
& \times\left[\begin{array}{ll}
(\gamma, q),\left(\frac{1}{2}+\psi+\xi, \eta\right),\left(\frac{1}{2}-\psi+\xi, \eta\right),(1,1) ; & \\
(\beta, \alpha),(1-\lambda+\xi, \eta),(\delta, 1) & \frac{\omega}{\phi^{\eta}}
\end{array}\right],
\end{aligned}
$$

\section{Special properties :}

(i) Putting $q=\delta=1$ in (4.5.2), we have

$$
\begin{aligned}
\int_{0}^{\infty} & \exp \frac{-\phi t}{2} t^{\xi-1} W_{\lambda, \psi}(\phi t) E_{\alpha, \beta}^{\gamma}\left(\omega t^{\eta}\right) d t \\
= & \frac{\phi^{-\xi}}{\Gamma(\gamma)} 3 \Psi_{2}\left[\begin{array}{ll}
(\gamma, q),\left(\frac{1}{2} \pm \phi+\xi, \eta\right) ; & \frac{\omega}{\phi^{\eta}} \\
(\beta, \alpha),(1-\lambda+\xi, \eta) ;
\end{array}\right],
\end{aligned}
$$

(ii) For $q=\gamma=\delta=1$ in (4.5.2), we have

$$
\begin{aligned}
\int_{0}^{\infty} \exp ^{\frac{-\phi t}{2}} t^{\xi-1} W_{\lambda, \psi}(\phi t) E_{\alpha, \beta}\left(\omega t^{\eta}\right) d t & \\
=\phi^{-\xi}{ }_{3} \Psi_{2} & {\left[\begin{array}{l}
\left(\frac{1}{2} \pm \phi+\xi, \eta\right),(1,1), ; \\
\frac{w}{\phi^{\eta}} \\
(\beta, \alpha),(1-\lambda+\xi, \sigma) ;
\end{array}\right] }
\end{aligned}
$$

(iii) Now putting $q=\beta=\alpha=\gamma=\delta=1$ in (4.5.2), we have

$$
\begin{aligned}
& \int_{0}^{\infty} \mathrm{e}^{\frac{-t}{2}} t^{\rho-1} W_{\lambda, \psi}(\phi t) \exp \left(\omega t^{\eta}\right) d t \\
& =\phi^{-\xi}{ }_{2} \Psi_{1}\left[\begin{array}{ll}
\left(\frac{1}{2} \pm \phi+\xi, \eta\right) ; & \\
(1-\lambda+\xi, \sigma) ; & \frac{w}{\phi^{\eta}}
\end{array}\right]
\end{aligned}
$$

\section{Relationship with some known special functions} Relationship with Wright hypergeometric function

If the condition (1.10) be satisfied, then (1.9) can be written as

$$
\begin{aligned}
& E_{\alpha, \beta, v, \sigma, \delta, p}^{\mu, \rho, \gamma, q}(z)=\sum_{n=0}^{\infty} \frac{(\mu)_{\rho n}(\gamma)_{q n} z^{n}}{\Gamma(\alpha n+\beta)(v)_{\sigma n}(\delta)_{p n}} \\
& =\frac{\Gamma(\delta) \Gamma(v)}{\Gamma(\mu) \Gamma(\gamma)} \sum_{n=0}^{\infty} \frac{\Gamma(\mu+\rho n) \Gamma(\gamma+q n) \Gamma(n+1)}{\Gamma(\alpha n+\beta) \Gamma(v+\sigma n) \Gamma(\delta+p n)} \frac{z^{n}}{n !}
\end{aligned}
$$

$$
=\frac{\Gamma(\delta) \Gamma(v)}{\Gamma(\mu) \Gamma(\gamma)}{ }_{3} \Psi_{3}\left[\begin{array}{l}
(\mu, \rho)(\gamma, q),(1,1), ; \\
(\beta, \alpha),(\nu, \sigma),(\delta, p) ;
\end{array}\right],
$$

\section{Relationship with Fox H-function}

Using (4.1.1), we have from

$$
\begin{aligned}
& E_{\alpha, \beta, \nu, \sigma, \delta, p}^{\mu, \rho, \gamma, q}(z) \\
& =\frac{\Gamma(\delta) \Gamma(v)}{2 \pi i \Gamma(\gamma)} \int_{L} \frac{\Gamma(s) \Gamma(1-s) \Gamma(\mu-\rho s) \Gamma(\gamma-q s)}{\Gamma(\beta-\alpha s) \Gamma(v-\sigma s) \Gamma(\delta-p s)}(-z)^{-s} d s \\
& =\frac{\Gamma(\delta) \Gamma(v)}{2 \pi i \Gamma(\gamma) \Gamma(\mu)} H_{3,3}^{1,3} \\
& \times\left[\begin{array}{l}
(0,1),(1-\mu, \rho),(1-\gamma, q) \\
(0,1)(1-\beta, \alpha),(1-v, \sigma),(1-\delta, p) ;
\end{array}\right]
\end{aligned}
$$

Relationship with generalized Laguerre polynomials

Putting $\alpha=k, \beta=\mu+1, \gamma=-m, q \in N$ with $q \mid m$ and replacing $\mathrm{z}$ by $z^{k}$ in (1.6), we get

$$
\begin{aligned}
& E_{k, \mu+1, \delta}^{-m, q}\left(z^{k}\right)=\sum_{n=0}^{\left[\frac{m}{q}\right]} \frac{(-m)_{q n} z^{k n}}{\Gamma(k n+\mu+1)(\delta)_{n}} \\
& =\sum_{n=0}^{\left[\frac{m}{q}\right]} \frac{(-1)^{q n} m !}{(m-q n) ! \Gamma(k n+\mu+1)} \frac{z^{k n}}{(\delta)_{n}} \\
& =\frac{\Gamma(m+1)}{\Gamma(k m+\mu+1)} \sum_{n=0}^{\left[\frac{m}{q}\right]} \frac{(-1)^{q n} \Gamma(k m+\mu+1)}{(m-q n) ! \Gamma(k n+\mu+1)} \frac{z^{k n}}{(\delta)_{n}} \\
& =\frac{\Gamma(m+1)}{\Gamma(k m+\mu+1)} Z_{\left[\frac{m}{q}\right], \delta}^{\mu}(z, k)
\end{aligned}
$$

where $Z_{\left[\frac{m}{q}\right], \delta}^{\mu}(z, k)$ is a generalization of $Z_{\left[\frac{m}{q}\right]}^{\mu}(z, k)$ given by Shukla et al (2007).

Note that $Z_{\left[\frac{m}{q}\right]}^{\mu}(z, k)$ is a polynomial of degree $\left[\frac{m}{q}\right]$ in $z^{k}$.

Further for $q=k=1, Z_{m, 1}^{\mu}(z, 1)=L_{m}^{\mu}(z)$, where $L_{m}^{\mu}(z)$ is a generalized Laguerre polynomial. So that

$$
E_{k, \mu+1,1}^{-m, q}(z)=\frac{\Gamma(m+1)}{\Gamma(k m+\mu+1)} L_{m}^{\mu}(z)
$$

which is the required relationship.

Competing interests

Authors declare that they have no competing interests.

\section{Authors' contributions}

Both the authors, viz. MAK and SA with the consultation of each other, carried out this work and drafted the manuscript together. Both the authors read and approved the final manuscript.

\section{Acknowledgements}

The authors wish to thank the refrees for valuable suggestions and comments. 


\section{References}

Mittag-Leffler GM (1903) Sur la nouvelle fonction $E_{\alpha}(x)$. CR Acad Sci Paris 137: 554-558

Prabhakar TR (1971) A singular integral equation with a generalised Mittag-Leffler function in the kernel. Yokohama Math.J 19: 7-15 Rainville ED (1960) Special Functions. Macmillan, New York

Saigo M, Kilbas AA (1998) On Mittag-Leffler type function and applications. Integral Transforms Spec Funct 7: 31-49

Salim TO (2009) Some properties relating to the generalized Mittag-Leffler function. Adv Appl Math Anal 4: 21-30

Salim TO, Ahmad W Faraj (2012) A generalization of integral operator associated with fractional calculus Mittag-Leffler function. J Fractional Calculus Appl 3(5): 1-13

Shukla AK, Prajapati JC (2007) On a generalised Mittag-Leffler function and its properties. J Math Anal Appl 336: 797-811

Sneddon IN (1979) The use of integral Transforms. Tata McGraw-Hill, New Delhi

Srivastava HM, Manocha HL (1984) A treatise on generating functions Wiley. Ellis Horwood Ltd., Chichester, England

Wiman A (1905) Uber den fundamental Satz in der Theorie der Funktionen $E_{\alpha}(x)$. Acta Math 29: 191-201

Whittaker ET, Watson GN (1962) A course of modern analysis. Cambridge Univ. Press, Cambridge

\section{doi:10.1186/2193-1801-2-337}

Cite this article as: Khan and Ahmed: On some properties of the generalized Mittag-Leffler function. SpringerPlus 2013 2:337.

\section{Submit your manuscript to a SpringerOpen ${ }^{\circ}$ journal and benefit from:}

- Convenient online submission

- Rigorous peer review

- Immediate publication on acceptance

- Open access: articles freely available online

- High visibility within the field

- Retaining the copyright to your article 